\title{
Influenza em pacientes com doenças cardíacas crônicas: o que há de novo?
}

\section{Influenza in patients with chronic heart diseases: what's new?}

Lucia Ferro Bricks ${ }^{1}$, Telma Regina Marques Pinto Carvalhanas ${ }^{2}$, Carla Magda Allan Santos Domingues ${ }^{3}$, Sirlene de Fátima Pereira $^{3}$, Nancy Cristina Junqueira Bellei ${ }^{4}$

1. Diretora Médica para Influenza na América Latina, Sanofi Pasteur. 2. Centro de Vigilância Epidemiológica da Secretaria de Saúde do Estado de São Paulo, (SP), Brasil. 3. Programa Nacional de Imunizações do Ministério da Saúde do Brasil (PNI/SVS/MS). 4. Docente do Departamento de Medicina da Universidade Federal de São Paulo (UNIFESP).

\section{Resumo}

Introdução: A influenza é uma doença infecciosa altamente transmissível, de distribuição global e que tem alto impacto na saúde pública. De acordo com a OMS, anualmente são registrados de três a cinco milhões de casos graves, que resultam em, aproximadamente, 0,25 a 0,5 milhão de mortes em todo o mundo. Indivíduos com doenças crônicas apresentam alto risco e complicações e morte e estão entre os grupos alvo para vacinação anual. Entretanto, as coberturas vacinais estão abaixo das preconizadas pela Assembleia Mundial da Saúde e OMS. Objetivo: Este texto é uma revisão não sistemática da literatura, com o objetivo de apresentar as informações mais recentes sobre os riscos da influenza e benefícios da vacina contra influenza em pacientes portadores de doenças cardíacas crônicas. Métodos: Levantamento dos artigos publicados sobre o tema no PUBMED, SCIELO e sites da OMS, PAHO e CDC, assim como as informações do Ministério da Saúde, no período de janeiro de 2000 a dezembro de 2014. Resultados: De um total de 143 artigos identificados sobre o tema, foram selecionadas 56 referências para esta revisão. Grande parte dos estudos, recentemente publicados, aponta para uma forte associação entre influenza e risco de complicações e morte por doença cardiovascular. Embora a vacina seja recomendada para cardiopatas, as coberturas vacinais são baixas na maioria dos países. Conclusões: É necessário conscientizar os profissionais de saúde e a população sobre os benefícios da vacinação.

Palavras-chave: Influenza. Vacinas. Cardiopatias.

\begin{abstract}
Introduction: Influenza is a highly transmissible infectious disease, distributed worldwide, that bears a heavy impact on public health. According to the WHO, a total of three to five million serious cases are reported annually. They lead to approximately 0.25 to 0.5 million deaths worldwide. Individuals with chronic diseases show a high risk of complications and death and are among the target groups for annual vaccination; nevertheless, the vaccine coverage is below that recommended by the World Health Assembly and the WHO. Objective: This paper is a non-systematic review of the literature that aims at presenting the most recent information on the risks of influenza and on the risks and benefits of the influenza vaccine in patients with chronic heart diseases. Methods: Survey of papers published on the subject in PUBMED, SCIELO and OMS, PAHO and CDC sites, as well as information from the Health Ministry, during the period from January 2000 to December 2014. Results: Out of a total of 143 articles found on the subject, 56 were selected for this review. Most studies recently published indicate a strong association between influenza and the risk of complications and death due to cardiovascular disease. Although influenza vaccine has been recommended for subjects with heart diseases, vaccine coverage is low in most countries. Conclusions: It is necessary to make health professionals and people aware of the vaccination benefits.
\end{abstract}

Keywords: Influenza. Vaccines. Heart diseases.

\section{INTRODUÇÃO}

A influenza é uma doença infecciosa altamente transmissível, de distribuição global, que tem alto impacto na saúde pública. De acordo com a OMS, anualmente são registrados três a cinco milhões de casos graves que resultam em, aproximadamente, 0,25 a 0,5 milhão de mortes em todo o mundo ${ }^{1}$. Indivíduos com doenças crônicas apresentam maior risco para complicações e morte por influenza e estão incluídos entre os grupos prioritários para vacinação. Desde 2003, a Assembleia Mundial da Saúde preconiza que as coberturas vacinais em grupos de risco sejam de, no mínimo, $75 \%^{2}$; no Brasil, a meta é atingir $80 \%$ de cobertura durante as campanhas anuais de vacinação ${ }^{3} \mathrm{e}$, nos EUA, o objetivo é mais ambicioso, tendo como meta atingir $90 \%$ de cobertura ${ }^{4}$. Apesar das recomendações para uso da vacina em grupos de risco, as coberturas em pessoas que apresentam doenças de base, em geral, são baixas ou desconhecidas s,5-12. $^{3}$.
Este artigo teve como objetivo analisar as informações mais recentes sobre os riscos da influenza e benefícios da vacinação de pacientes portadores de doenças cardíacas crônicas.

\section{MÉTODOS}

Este artigo faz uma revisão não sistemática da literatura sobre os riscos da influenza em pessoas que apresentam doenças crônicas e os benefícios da vacinação em portadores de doenças cardíacas crônicas. Para tal, foi realizado um levantamento dos artigos publicados sobre o tema no PUBMED, SCIELO e sites da Organização Mundial de Saúde (OMS), Organização Pan-americana de Saúde (OPAS) e Centro de Controle de Doenças (CDC) dos Estados Unidos da América (EUA), assim como das informações do Ministério da Saúde, no período de

Correspondência: Lucia Ferro Bricks. Divisão Vacinas do Grupo Sanofi, Brasil. Av. Sylvio de Magalhães Padilha, 5200. Jardim Morumbi. CEP: $01246-902$. São Paulo, SP. E-mail: lfbricks@gmail.com

Conflito de interesse: Lucia Ferro Bricks é Diretora Médica para Influenza da Sanofi Pasteur, América Latina. Não há conflito de interesse por parte dos outros autores

Recebido em: 14 Setembro 2015; Revisado em: 19 Setembro 2015; Aceito em: 25 Setembro 2015. 
janeiro de 2000 a dezembro de 2014, usando como palavras chave: influenza, vacinas, e doenças cardíacas crônicas. Foram excluídos os textos que focam a vacinação de idosos, gestantes e crianças menores de cinco anos, assim como aqueles que se referiam exclusivamente a outras patologias (metabólicas, pulmonares, renais, hepáticas, imunodeficiências).

\section{RESULTADOS}

Para esta revisão, foram inicialmente revisados 173 artigos, sendo selecionados os mais relevantes (meta-análises, estudos prospectivos e ecológicos), além das informações mais recentes sobre influenza e doenças cardíacas crônicas encontradas nos sites consultados.

\section{Riscos da influenza em pacientes portadores de doenças crônicas}

A relação entre o aumento da circulação dos vírus influenza e as mortes por pneumonia está bem estabelecida há décadas ${ }^{1,13-15}$. Em um estudo sobre a elevada mortalidade observada nos EUA em 26 estações (1970-1975), em média, o elevado índice de hospitalizações por pneumonia e influenza (P\&I) foi de 49 por 100.000 pessoas (variação 8 a 102/100.000), mas durante as epidemias em que predominou a cepa $A(H 3 N 2)$, o risco dobrou em comparação com as epidemias causadas pelas cepas A(H1N1) e B sazonais. Embora o risco de hospitalização por P\&I seja mais elevado em idosos (174/100.000) em comparação com pessoas com menos de 65 anos (33/100.000), o grupo mais jovem é responsável por mais da metade do número total de hospitalizações associadas à $P \& \mid$ e, durante as pandemias, observou-se desvio da faixa etária, com maior número de mortes em adultos jovens ${ }^{14}$. Este fato também foi observado após o surgimento da cepa pandêmica causada pelo vírus $\mathrm{A}(\mathrm{H} 1 \mathrm{~N} 1) \mathrm{pdm} 09^{12,16-20}$.

Em estudo recentemente publicado, verificou-se que a infecção por influenza dobra o risco de pneumonia por Streptococcus pneumoniae (OR=2,2 IC95\%: 1,1-4,5) e que o risco é ainda maior nos pacientes colonizados por essa bactéria $(\mathrm{OR}=8,2$; IC95\%: $2,7-25,0)^{15}$. Apesar de a relação entre infecção por influenza e pneumonias estar muito bem estabelecida, é importante lembrar que a infecção é sistêmica e que os vírus podem afetar vários órgãos, exacerbar doenças crônicas subjacentes e causar diversos tipos de complicações cardiovasculares, metabólicas, neurológicas e renais. A maior gravidade é observada em pessoas com doenças subjacentes, nos extremos de idade (idosos e crianças menores de cinco anos) e em gestantes ${ }^{16,21,22}$.

Em recente revisão sistemática e meta-análise de 234 artigos sobre os riscos de morte por influenza em diversos tipos de doenças crônicas $^{23}$, verificou-se que a presença de qualquer fator de risco aumenta o risco de morte por influenza entre 2 e 2,7 vezes, respectivamente, para influenza sazonal e pandêmica. As pneumonias que se seguem à infecção por influenza são muito comuns e também predispõe a complicações cardíacas. A incidência de qualquer complicação cardíaca após pneumonias adquiridas na comunidade (PAC) foi estimada em 17,7\%; a incidência de insuficiência cardíaca pós-PAC foi $14,1 \%$ e as incidências de síndromes coronarianas agudas e arritmias cardíacas, 5,3\% e 4,7\%, respectivamente ${ }^{24}$.

Estima-se que as doenças cardiovasculares (DCV) sejam responsáveis por $20 \%$ das mortes no mundo, atingindo cifras de $50 \%$ nos países desenvolvidos ${ }^{25}$. Em 2010, as DCV foram a principal causa de morte em todo o mundo. Embora sua incidência aumente com a idade, em pessoas que vivem em países de baixa ou média renda, o primeiro episódio de angina se manifesta, em média, aos 55 anos de idade e muitos portadores de fatores de risco de infarto agudo do miocárdio (IAM) não estão cientes de sua condição de risco ${ }^{26}$.

Nos EUA, estima-se que, em 2010, a prevalência de DCV tenha sido de $35,3 \%$ (83,6 milhões de casos) e a prevalência de doença cardíaca coronariana (DCC), 6,4\% (15,4 milhões de casos), com um total de 7,6 milhões de infartos agudos do miocárdio (IAM), 7,8 milhões de angina pectoris. As doenças coronarianas causaram uma em cada seis mortes em 2010, com um total de 379.059 mortes. A cada ano, aproximadamente, 620.000 americanos têm novo ataque cardíaco (primeira hospitalização por IAM ou DCC) e, aproximadamente, 250.000 têm infarto recorrente. Adicionalmente, 150.000 pessoas têm "infarto silencioso" todos os anos. A cada 34 segundos, um americano apresenta evento coronariano e a cada um minuto e 23 segundos, ocorre uma morte por doença cardíaca ${ }^{27}$. A prevalência de DCV, assim como de diversas outras condições crônicas, como doença pulmonar obstrutiva crônica (DPOC) e diabetes, aumenta com a idade e, frequentemente, essas doenças são subdiagnosticadas. A análise das informações sobre 5.270 adultos americanos hospitalizados com infecção confirmada por influenza sazonal entre 2005 e 2009 e de 4.962 adultos internados com infecção confirmada pela cepa pandêmica $A(H 1 N 1) p d m 09$ entre 2009 e 2010, revelou que as principais condições crônicas associadas às cepas sazonais foram doença cardiovascular crônica (41,9\%), doença pulmonar crônica $(37,7 \%)$ e doenças metabólicas crônicas (40,9\%), com destaque para diabetes (26,7\%); entre os casos hospitalizados por influenza pandêmica, a maior frequência de hospitalização foi registrada em portadores de doenças pulmonares crônicas $(42,5 \%)$, seguida por doenças metabólicas crônicas $(31,9 \%)$ e doenças cardiovasculares $(20,1 \%)$. As taxas de hospitalização em unidades de cuidados intensivos e necessidade de ventilação mecânica foram significativamente mais altas em adultos com infecção pela cepa pandêmica ( 23,4 vs $15,3 \%$ e 12,9 vs $8,9 \%)$, mas as taxas de letalidade não foram significativamente diferentes, variando entre 3,6 e $5,0 \%{ }^{16}$.

No Brasil, os dados da pesquisa nacional de Saúde 2013, indicam que $4,2 \%$ das pessoas com mais de 18 anos de idade $(6,1$ milhões) têm alguma doença cardíaca. A proporção aumenta significativamente com a idade, sendo de $0,9 \%$ em pessoas com idade entre 18 e 29 anos ; 3,4\% entre 30 e 59 anos; $11,9 \%$ entre 65 e 74 anos e $13,7 \%$ nos maiores de 75 anos. A angina de grau 2 (pessoas que apresentam dor ou desconforto ao caminhar em lugar plano e com velocidade normal) foi estimada em 4,2\% em pessoas com mais de 18 anos, enquanto a angina de grau 1 
(presença de dor ou desconforto ao subir ladeiras ou um lance de escadas) foi de $7,6 \%^{28}$.

Em 2013, a maioria das mortes comprovadas por influenza no Brasil foi causada pela cepa $\mathrm{A}(\mathrm{H} 1 \mathrm{~N} 1)$ pdm09 e confirmada em adultos jovens (20-60) portadores de doenças crônicas cardíacas, pulmonares e metabólicas ${ }^{17}$. Em 2014, a cepa A(H3N2) foi a predominante, e a maioria das mortes foi registrada em idosos; as doenças cardiovasculares e pulmonares ficaram, respectivamente, em segundo e terceiro lugares ${ }^{18,19}$

\section{Relação entre infecção por influenza e aumento na incidência de eventos cardiovasculares}

A possível relação entre influenza e IAM foi primeiramente observada na Europa e nos EUA logo após a identificação dos vírus $(1900 s)^{13}$. Posteriormente, diversas publicações mostraram que a incidência de eventos cardiovasculares aumenta durante os períodos em que existe maior circulação dos vírus influenza ${ }^{29-31}$.

Os possíveis mecanismos associados ao aumento de complicações cardiovasculares durante a estação de influenza podem resultar de efeitos diretos dos vírus influenza sobre o miocárdio, causando miocardite, ou da resposta inflamatória, que leva à exacerbação de doenças crônicas preexistentes. As manifestações clínicas das infecções por vírus influenza A e B são indistinguíveis ${ }^{32,33}$ e ambos os tipos podem causar complicações cardíacas; entretanto, aparentemente, as cepas $A(H 3 N 2)$ apresentam maior impacto na mortalidade de idosos; a cepa pandêmica $A(H 1 N 1) p d m 09$, acomete predominantemente adultos com comorbidades e as cepas $B$ acometem desproporcionalmente pessoas com menos de 18 anos de idade, sendo relevante causa de morte na faixa etária pediátrica ${ }^{34}$. No Brasil, dados da Secretaria de Estado da Saúde do Estado de São Paulo revelaram que de 64 a 76\% das mortes confirmadas por influenza em 2013 e 2014 foram registradas em pessoas com menos de 60 anos de idade. Também ficou evidente que as doenças cardíacas e pulmonares crônicas estavam entre as mais frequentes comorbidades associadas às infecções graves e mortes e que, embora todas as cepas possam causar infecções graves e mortes em pessoas de qualquer idade, as cepas $A(\mathrm{H} 1 \mathrm{~N} 1)$ e $B$ têm impacto desproporcional nos casos graves e mortes em menores de 60 anos $^{19,20}$.

As mortes por DCV, especialmente IAM, são mais comuns no inverno do que no verão e, embora muitos fatores possam contribuir para o aumento da mortalidade por IAM no inverno, as infecções respiratórias parecem desempenhar papel relevante nesse aumento da mortalidade e, entre elas, a influenza merece destaque ${ }^{35-39}$.

No Reino Unido, entre 20.486 pessoas com primeiro IAM e 19.063 com primeiro acidente vascular cerebral (AVC), verificouse que o risco de ambos os eventos aumentou após infecções respiratórias. O risco de infarto do miocárdio foi quase cinco vezes maior (OR $=4,95$; IC95\% 4,43-5,53) e o de AVC, três vezes maior (OR =3,19; IC95\%: 2,81-3,62) nos primeiros três dias após J. Health Biol Sci. 2015; 3(3):165-171 o início da infecção, caindo posteriormente. A vacinação contra influenza reduziu significativamente a ocorrência de eventos vasculares $^{35}$. Em um estudo caso-controle com 11.155 casos de primeiro infarto do miocárdio e 9.208 casos de AVC, encontrouse forte associação entre antecedente de infecção respiratória aguda na semana anterior a ambos os eventos, com queda no risco com o passar do tempo. O risco de ambos os eventos, praticamente dobrou até sete dias após o início da infecção respiratória ${ }^{36}$.

Nos EUA, em estudo com 233 casos e 363 controles com idade entre 21 e 89 anos, realizado em Los Angeles, verificou-se que o antecedente de infecção respiratória aguda dobrou o risco de eventos tromboembólicos e quadruplicou o risco em pacientes sem fatores de risco cardiovascular (OR=4,15; IC95\%: 1,2214,1). Os autores concluíram que as infecções respiratórias agudas agem disparando ou aumentando o risco de eventos vasculares, especialmente entre pessoas sem doença vascular prévia. Neste estudo, mais da metade dos casos e controles tinha menos de 57 anos $^{37}$.

Embora nos estudos acima mencionados não tenha sido possível isolar os agentes etiológicos das infecções respiratórias agudas, a vigilância das mortes por pneumonia e influenza em idosos, realizada nos EUA entre 1959 e 1999, revelou claramente uma relação entre mortes e períodos com maior circulação dos vírus influenza ${ }^{38}$. As incidências de IAM e de infecção por vírus influenza também seguem o mesmo padrão sazonal em outros países, incluindo o Brasil ${ }^{24,39-43}$.

Embora as manifestações clínicas e as lesões histopatológicas associadas aos vírus influenza $A$ e $B$ sejam indistinguíveis, em estudo conduzido nos EUA em que foram analisados 45 casos de infecção fatal confirmada pelo vírus influenza $B$, a evolução fatal foi muito mais rápida nos casos de infecção por vírus $B$ (metade dos pacientes morreu dentro de três dias após o início dos sintomas e, aproximadamente, $70 \%$ dentro de quatro dias). Além disso, em apenas $24 \%$ dos pacientes menores de 18 anos de idade houve associação com pneumonia bacteriana ${ }^{34}$. Esses dados são muito importantes, tendo em vista que raramente se suspeita dos vírus influenza em casos de morte sem pneumonia concomitante $\mathrm{e}^{14,34,44}$.

\section{Impacto da vacinação contra influenza em doenças cardiovasculares}

Embora as complicações cardíacas associadas à infecção por influenza já sejam bem conhecidas, o impacto da vacinação na prevenção de mortes ainda é objeto de controvérsias ${ }^{41}$. Grande parte dos estudos, entretanto, revela que a vacinação contra influenza reduz a mortalidade neste grupo ${ }^{35,36,45}$. A aterosclerose é uma doença inflamatória e a vacinação contra influenza, além de prevenir a infecção, reduz o risco de eventos cardiovasculares associados à doença, possivelmente por reduzir a inflamação sistêmica associada à leucocitose e à redução da albumina que, por sua vez, alteram a função do endotélio, causam instabilidade do ateroma e a subsequente rotura das placas. Outros possíveis mecanismos fisiopatológicos podem estar 
associados às alterações nos fatores de coagulação, aumento na produção de citocinas e inibição na função vasodilatadora do óxido nítrico ou prostaglandinas ${ }^{13,29,37,46}$.

O primeiro estudo caso-controle para avaliar o impacto da vacinação contra influenza na redução de infarto do miocárdio (IM) foi publicado no ano 2000 e incluiu 218 pacientes com doença cardiovascular atendidos no Memorial Hermann Hospital, na estação de influenza 1997/1998, nos EUA. Pacientes que apresentaram IM ( $\mathrm{N}=109)$ foram selecionados como casos e os que não tiveram IM ( $N=109)$, como controles. Não houve diferença entre idade média dos casos e controles $(62,9$ vs. 64,6 anos) e a maioria era do sexo masculino $(61 \%$ e 65\%). Hipertensão, hipercolesterolemia e fumo foram significativamente associados à ocorrência de $\mathrm{IM}$, enquanto a vacinação contra influenza reduziu o risco IM em 67\% (OR = 0,33; $\mathrm{Cl} 95 \%$ : 0,12-0,82) ${ }^{13}$.

Em outro estudo realizado nos EUA, foram incluídos 200 pacientes com IM admitidos nas primeiras 72 horas e 101 pacientes que seriam submetidos à angioplastia, sem apresentar doença coronariana instável, cirurgia prévia (Bypass, angioplastia) ou evidência de necrose tissular. Ambos os grupos foram alocados para receber, ou não, a vacina influenza e a análise de morte, re-infarto, re-hospitalização por isquemia foram avaliadas nos seis meses seguintes. A morte ocorreu em $2 \%$ dos vacinados vs. $8 \%$ dos controles ( $R R=0,25$; IC $95 \% 0,07$ $0,86 ; P=0,010)$. A ocorrência de qualquer evento foi verificada em $11 \%$ dos vacinados vs. $23 \%$ dos controles $(P=0,009)$. $A$ vacinação reduziu o risco de morte e eventos isquêmicos em pacientes que sofreram infarto e naqueles que se recuperaram de angioplastia durante a estação de influenza ${ }^{29}$.

Em Taiwan, a vacinação contra influenza reduziu as taxas de hospitalização por todas as causas em pacientes com e sem condições de risco em $20 \%$ e $23 \%$, respectivamente. A redução nas hospitalizações por doenças pulmonares e cardíacas foi $42 \%$ e $32 \%$, respectivamente. Além disso, nos pacientes vacinados, o tempo médio de hospitalização foi reduzido, em média, em 2,4 dias. O benefício foi maior (4,9 dias) quando a hospitalização estava associada a doenças pulmonares ${ }^{31}$.

Nos EUA, durante 1998-1999 e 1999-2000, os dados administrativos e clínicos de pacientes com mais de 65 anos seguidos em três grandes organizações de saúde revelaram que a vacinação contra influenza foi associada com a redução no risco de hospitalização por doenças cardíacas em $19 \%$ nas duas estações. A redução de doença cerebrovascular foi de $16 \%$ na primeira e de $23 \%$ na segunda estação. A redução na incidência de pneumonia ou influenza foi maior (29\% a $32 \%$ ) e o risco de morte caiu quase pela metade ${ }^{30}$.

Em meta-análise em que foram revisados os dados de cinco estudos publicados e um estudo não publicado entre 1946 e agosto de 2013, incluindo 6.735 pacientes seguidos, em média por 7,9 meses, concluiu-se que a vacinação contra influenza reduziu o risco de morte por eventos cardiovasculares em $36 \%$ $(R R=0,64 ;$ IC95\% 0,48-0,86), propiciando maiores benefícios aos pacientes que apresentavam doença coronariana ativa ${ }^{21}$.

Estudos mais recentemente publicados e não incluídos nessa meta-análise também confirmam os benefícios da vacinação $0^{47-50}$.

$\mathrm{Na}$ Austrália, em um estudo caso-controle com 559 participantes atendidos em um hospital terciário entre 2008 e 2010, verificou-se que a efetividade da vacina influenza em reduzir as taxas de IAM foi de $45 \%$ (OR $=0,55$; IC95\%: 0,35$0,85)$. Embora o antecedente de infecção por influenza não tenha sido reconhecido como fator de risco para IAM, em quase $10 \%$ dos pacientes hospitalizados, não foi aventada suspeita de infecção por influenza ou confirmada por testes laboratoriais. A vacinação reduziu significativamente as taxas de IAM e os autores concluíram que ela poderia beneficiar particularmente as pessoas do grupo etário entre 50 e 64 anos com fatores de risco por doenças cardiovasculares que não são incluídas no grupo-alvo da vacinação ${ }^{47}$.

Em Israel, o acompanhamento durante um ano de 1.964 pacientes ( $\geq 50$ anos) com insuficiência cardíaca, revelou que a vacinação contra influenza reduziu a hospitalização e a mortalidade em $29 \%$ e $19 \%$, respectivamente. Em quatro anos de seguimento, a mortalidade caiu $17 \%{ }^{48}$.

Na Coreia, a análise dos resultados de um estudo multicêntrico com 828 pacientes hospitalizados por exacerbação de asma, DPOC, doença cardíaca isquêmica ou insuficiência cardíaca congestiva (casos) e 828 controles atendidos em ambulatório entre dezembro de 2011 e maio de 2012 revelou que a vacinação reduziu em $1 / 3(33,7 \%)$ o risco de hospitalização. Maiores benefícios foram observados em idosos (> 65 anos), grupo em que a efetividade foi de $56 \%{ }^{49}$.

No Brasil, após o início das campanhas de vacinação de idosos contra influenza, verificou-se redução acentuada no número de mortes por doenças cardíacas isquêmicas e doenças cerebrovasculares, mas não nas mortes por causas externas, no período de 1980 a $2006^{43}$.

\section{Coberturas vacinais em pacientes portadores de doenças crônicas}

Apesar das recomendações para vacinar adultos com doenças de base, incluindo doenças cardíacas, as coberturas vacinais nesses grupos têm sido baixas em todos os países ${ }^{1-10,51}$.

Em um e estudo realizado na Europa com 16.913 adultos de 11 países, verificou-se que as coberturas vacinais nos grupos de risco variaram muito entre os países, sendo maiores na Holanda $(75 \%)$ e mais baixas na Grécia $(27 \%)^{9}$. O aumento nas coberturas vacinais em grupos de risco poderia trazer enorme impacto na saúde pública ${ }^{9,52,53}$.

Nos EUA, na estação de 2013/2014, 37\% dos adultos hospitalizados por influenza tinham doença cardíaca, mas, assim como na Europa, as coberturas vacinais em grupos de risco com menos de 60 anos deixam a desejar ${ }^{53}$. 
No Brasil, em 2013 e 2014, as doenças cardíacas foram identificadas em, aproximadamente, $1 / 3$ das mortes confirmadas por influenza ${ }^{17,18}$. A maioria dos óbitos confirmados por influenza em pessoas com menos de 60 anos de idade apresentava pelo menos uma doença crônica e não havia sido vacinada contra influenza, apesar de a vacina estar disponível gratuitamente nas unidades de saúde durante as campanhas de vacinação ${ }^{17-20}$.

A recomendação para vacinação por parte de médicos e outros profissionais de saúde é o mais forte fator associado ao aumento das coberturas vacinais e deve ser estimulada, considerando-se que as vacinas inativadas são seguras e efetivas ${ }^{54}$. Vale ressaltar que a vigilância da influenza se baseia na investigação dos casos de síndrome gripal e síndrome respiratória aguda grave e que grande parte das complicações e mortes ocorre mais de uma semana após o início da infecção; portanto, o real impacto da doença é subestimado, particularmente, entre pacientes que desconhecem ser portadores de enfermidades crônicas ${ }^{55,56}$.

\section{CONCLUSÃO}

Tanto a influenza como as doenças cardiovasculares são causa importante de morte em todo o mundo. Existem evidências de que a influenza pode causar miocardite, arritmias cardíacas e desencadear infarto agudo do miocárdio, causando grande número de complicações graves e mortes, mesmo em pessoas previamente saudáveis. A vacinação contra influenza é particularmente indicada para grupos de risco de complicações, como pessoas que apresentam doenças cardíacas, mas as coberturas vacinais são baixas. Nesse sentido, o papel dos profissionais de saúde recomendando a vacinação em pacientes com doenças cardíacas crônicas é de suma relevância, tendo em vista que as evidências atuais indicam que a vacinação pode reduzir significativamente a mortalidade em pacientes com doenças cardíacas pré-existentes.

\section{REFERÊNCIAS}

1. World Health Organization. Vaccines against influenza WHO position paper November 2012.Wkly Epidemiol Rec. 2012 Nov 23;87(47):461-76.

2. World Health Organization. Resolution of the World Health Assembly. Prevention and control of influenza pandemics and annual epidemics [Internet] In: 56thWorld Heath Assembly; 2003 May 19-28 [cited 2015 Jul 5]; Geneva, Switzerland. Available from: http://apps.who.int/gb/archive/pdf_files/WHA56/ ea56r19.pdf.

3. Domingues CM, de Oliveira WK. Uptake of pandemic influenza (H1N1)-2009 vaccines in Brazil, 2010. Vaccine. 2012 Jul 6;30(32):4744-51. doi: 10.1016/j. vaccine.2012.05.007. PubMed PMID: 22609010.

4. Washington. Department of Health and Human Services. Healthy People 2020. Immunization and infectious diseases: objectives, IID -12 [Internet]. 2014 [updated 2014 Aug 11; cited 2014 Jul 28]. Available from: http://healthypeople. gov/2020/topicsobjectives2020/objectiveslist.aspx?topicid=23.

5. Ropero-Alvarez AM. Influenza vaccine use in the Americas: network for evaluation of influenza vaccine effectiveness: REVELAC-i [Internet]. In Comprehensive Family Immunization Unit; 2014 July 1-2; Washington. [cited 2014 Dec 5]. Available from: http://www.paho.org/hq/index.php?option=com docman\&task=doc_view\&gid=27002\&Itemid=270\&lang=fr.

6. Michiels B, Govaerts F, Remmen R, Vermeire E, Coenen S. A systematic review of the evidence on the effectiveness and risks of inactivated influenza vaccines in different target groups. Vaccine. 2011 Nov 15;29(49):9159-70. doi: 10.1016/j. vaccine.2011.08.008. PubMed PMID: 21840359.

7. World Health Organization. Meeting of the Strategic Advisory Group of Experts on immunization, April 2012 - conclusions and recommendations. Wkly Epidemiol Rec. 2012 May 25;87(21):201-16.

8. Centers for Disease Control and Prevention. Prevention and control of seasonal influenza with vaccines. Recommendations of the Advisory Committee on Immunization Practices--United States, 2013-2014. MMWR Recomm Rep. 2013 Sep 20;62(RR-07):1-43.

9. Preaud E, Durand L, Macabeo B, Farkas N, Sloesen B, Palache A, et al. Annual public health and economic benefits of seasonal influenza vaccination: a European estimate. BMC Public Health. 2014 Aug 7;14:813. doi: 10.1186/14712458-14-813. PubMed PMID: 25103091.

10. Loerbroks A, Stock C, Bosch JA, Litaker DG, Apfelbacher CJ. Influenza vaccination coverage among high-risk groups in 11 European countries. Eur J Public Health. 2012 Aug;22(4):562-8. doi: http://dx.doi.org/10.1093/eurpub/ ckr094.
11. Lu PJ, Gonzalez-Feliciano A, Ding H, Bryan LN, Yankey D, Monsell EA, et al. Influenza A (H1N1) 2009 monovalent and seasonal influenza vaccination among adults 25 to 64 years of age with high-risk conditions--United States, 2010. Am J Infect Control. 2013 Aug;41(8):702-9. doi: 10.1016/j.ajic.2012.10.027. PubMed PMID: 23419613.

12. Brasil. Ministério da Saúde. SI-PNI - Sistema de Informação do Programa Nacional de Imunizações. Campanha Nacional de vacinação contra gripe2014 [Internet]. 2014 [citado 2015 Mar 10]. Disponível em: http://pni.datasus.gov.br/consulta_Influenza_14_selecao. asp?enviar=ok\&sel=vacinometro\&faixa=todos\&grupo=todos\&uf $=$.

13. Naghavi M, Barlas Z, Siadaty S, Naguib S, Madjid M, Casscells W. Association of influenza vaccination and reduced risk of recurrent myocardial infarction. Circulation. 2000 Dec 19;102(25):3039-45. PMID: 11120692.

14. Simonsen L, Fukuda K, Schonberger LB, Cox NJ. The impact of influenza epidemics on hospitalizations. J Infect Dis. 2000 Mar;181(3):831-7. PMID: 10720501.

15. Wolter N, Tempia S, Cohen C, Madhi SA, Venter M, Moyes J, et al. High nasopharyngeal pneumococcal density, increased by viral coinfection, is associated with invasive pneumococcal pneumonia. J Infect Dis. 2014 Nov 15;210(10):1649-57. doi: 10.1093/infdis/jiu326. PubMed PMID: 24907383.

16. Reed C, Chaves SS, Perez A, D'Mello T, Daily Kirley P, Aragon D, et al. Complications Among Adults Hospitalized With Influenza: A Comparison of Seasonal Influenza and the 2009 H1N1 Pandemic. Clin Infect Dis. 2014 Jul 15;59(2):166-74. doi: 10.1093/cid/ciu285. PubMed PMID: 24785230.

17. Ministério da Saúde (Brasil). Secretaria de Vigilância de Saúde. Boletim epidemiológico. Influenza: monitoramento até a semana epidemiológica 52 de 2013 [Internet]. 2013 [acesso em: 2014 Jul 04]. Disponível em: http://u.saude. gov.br/images/pdf/2014/maio/22/boletim-influenza-se52de2013-220514.pdf.

18. Ministério da Saúde (Brasil). Secretaria de Vigilância em Saúde. Boletim epidemiológico. Influenza: Monitoramento até a Semana Epidemiológica 53 de 2014 [Internet]. 2014 [acesso em: 2015 Abr 15]. Disponível em: http:// portalsaude.saude.gov.br/images/pdf/2015/fevereiro/06/Boletim-Epidemiol-gico-Influenza-SE53-2014.pdf.

19. Carvalhanas TRMP, Ferreira PM, Pinto FKA, Yu ALF, Paiva TM, Benega MA, et al. Comorbidities identified in 616 influenza confirmed SARI inpatient cases and 116 deaths reported in São Paulo State (SP), Brazil, 2014 [abstract]. In: 33rd Annual Meeting of the European Society for Paediatric Infectious Diseases; 2015 May 12-16. Leipzig, Germany. 
20. Carvalhanas TRMP, Ferreira PM, Yu ALF, Pinto FKA, Paiva TM, Benega MA et al. Review of 3,310 influenza serious acute respiratory infections (sari) and 567 deaths reported in São Paulo State (SP), Brazil, 2013-2014 [abstract]. In: 33rd Annual Meeting of the European Society for Paediatric Infectious Diseases; 2015 May 12-16. Leipzig, Germany.

21. Udell JA, Zawi R, Bhatt DL, Keshtkar-Jahromi M, Gaughran F, Phrommintiku $A$, et al. Association between influenza vaccination and cardiovascular outcomes in high-risk patients: a meta-analysis. JAMA. 2013 Oct 23;310(16):1711-20. doi:10.1001/jama.2013.279206.

22. Estabragh ZR, Mamas MA. The cardiovascular manifestations of influenza: a systematic review. Int J Cardiol. 2013 Sep 10;167(6):2397-403. doi: 10.1016/j. ijcard.2013.01.274. PubMed PMID: 23474244.

23. Mertz D, Kim TH, Johnstone J, Lam PP, Science M, Kuster SP, et al. Populations at risk for severe or complicated influenza illness: systematic review and metaanalysis. BMJ. 2013 Aug 23;347:f5061. doi: http://dx.doi.org/10.1136/bmj f5061.

24. Corrales-Medina VF, Suh KN, Rose G, Chirinos JA, Doucette S, Cameron DW et al. Cardiac complications in patients with community-acquired pneumonia: a systematic review and meta-analysis of observational studies. PLoS Med. 2011 Jun;8(6):e1001048. doi: 10.1371/journal.pmed.1001048. PubMed PMID 21738449 .

25. Gurfinkel E, Lernoud V. The role of infection and immunity in atherosclerosis Expert Rev Cardiovasc Ther. 2006 Jan;4(1):131-7. PubMed PMID: 16375635.

26. Moran AE, Forouzanfar MH, Roth GA, Mensah GA, Ezzati M, Flaxman A, et al. The global burden of ischemic heart disease in 1990 and 2010: the Global Burden of Disease 2010 study. Circulation. 2014 Apr 8;129(14):1493-501. doi 10.1161/CIRCULATIONAHA.113.004046. PubMed PMID: 24573351.

27. Go AS, Mozaffarian D, Roger VL, Benjamin EJ, Berry JD, Blaha MJ, et al. Executive summary: heart disease and stroke statistics--2014 update: a report from the American Heart Association. Circulation. 2014 Jan 21;129(3):399-410. doi; 10.1161/01.cir.0000442015.53336.12. PubMed PMID: 24446411.

28. Ministério da Saúde (Brasil). Departamento de Análise de Situação de Saúde. Pesquisa Nacional de Saúde 2013. Percepção do estado de saúde, estilos de vida e doenças crônicas: Brasil, grandes regiões e Unidades da Federação [Internet]. 2014 [acesso em: 2015 Jul 5]. Disponível em: http://biblioteca.ibge. gov.br/visualizacao/livros/liv91110.pdf.

29. Gurfinkel EP, de la Fuente RL, Mendiz O, Mautner B. Influenza vaccine pilot study in acute coronary syndromes and planned percutaneous coronary interventions: the FLU Vaccination Acute Coronary Syndromes (FLUVACS) Study. Circulation. 2002 May 7;105(18):2143-7. PubMed PMID: 11994246.

30. Nichol KL, Nordin J, Mullooly J, Lask R, Fillbrandt K, Iwane M. Influenza vaccination and reduction in hospitalizations for cardiac disease and stroke among the elderly. N Engl J Med. 2003 Apr 3;348(14):1322-32. doi: 10.1056/ NEJMoa025028.

31. Wang CS, Wang ST, Lai CT, Lin LJ, Lee CT, Chou P. Reducing major cause-specific hospitalization rates and shortening hospital stays after influenza vaccination. Clin Infect Dis. 2004 Dec 1;39(11):1604-10. PubMed PMID: 15578359.

32. Su S, Chaves SS, Perez A, D'Mello T, Kirley PD, Yousey-Hindes K, et al. Comparing clinical characteristics between hospitalized adults with laboratoryconfirmed influenza a and B virus infection. Clin Infect Dis. 2014 Jul 15;59(2):252 5. doi:10.1093/cid/ciu269. PubMed PMID: 24748521.

33. Daley AJ, Nallusamy R, Isaacs D. Comparison of influenza A and influenza $B$ virus infection in hospitalized children. J Paediatr Child Health. 2000 Aug;36(4):332-5. PubMed PMID: 10940165.

34. Paddock CD, Liu L, Denison AM, Bartlett JH, Holman RC, Deleon-Carnes M, et al. Myocardial injury and bacterial pneumonia contribute to the pathogenesis of fatal influenza B virus infection. J Infect Dis. 2012 Mar 15;205(6):895-905. doi: 10.1093/infdis/jir861. PubMed PMID: 22291193.
35. Smeeth L, Thomas SL, Hall AJ, Hubbard R, Farrington P, Vallance P. Risk of myocardial infarction and stroke after acute infection or vaccination. $\mathrm{N} \mathrm{Engl} \mathrm{J}$ Med. 2004 Dec 16;351(25):2611-8. PubMed PMID: 15602021.

36. Clayton TC, Thompson M, Meade TW. Recent respiratory infection and risk of cardiovascular disease: case-control study through a general practice database. Eur Heart J. 2008 Jan;29(1):96-103. PubMed PMID: 18063596.

37. Paganini-Hill A, Lozano E, Fischberg G, Perez Barreto M, Rajamani K, Ameriso SF, et al. Infection and risk of ischemic stroke: differences among stroke subtypes. Stroke. 2003 Feb;34(2):452-7. PubMed PMID: 12574559.

38. Reichert TA, Simonsen L, Sharma A, Pardo SA, Fedson DS, Miller MA Influenza and the winter increase in mortality in the United States, 1959-1999. Am J Epidemiol. 2004 Sep 1;160(5):492-502. PubMed PMID: 15321847.

39. Foster ED, Cavanaugh JE, Haynes WG, Yang M, Gerke AK, Tang F, et al. Acute myocardial infarctions, strokes and influenza: seasonal and pandemic effects. Epidemiol Infect. 2013 Apr;141(4):735-44. doi: 10.1017/S0950268812002890. PubMed PMID: 23286343.

40. Warren-Gash C, Bhaskaran K, Hayward A, Leung GM, Lo SV, Wong CM. Circulating influenza virus, climatic factors, and acute myocardial infarction: a time series study in England and Wales and Hong Kong. J Infect Dis. 2011 Jun 15;203(12):1710-8. doi: 10.1093/infdis/jir171. PubMed PMID: 21606529.

41. Warren-Gash C, Smeeth L, Hayward AC. Influenza as a trigger for acute myocardial infarction or death from cardiovascular disease: a systematic review. Lancet Infect Dis. 2009 Oct;9(10):601-10. PubMed PMID: 19778762.

42. Madjid M, Miller CC, Zarubaev VV, Marinich IG, Kiselev OI, Lobzin YV, et al. Influenza epidemics and acute respiratory disease activity are associated with a surge in autopsy-confirmed coronary heart disease death: results from 8 years of autopsies in 34,892 subjects. Eur Heart J. 2007 May;28(10):1205-10. PubMed PMID: 17440221.

43. Mansur Ade $P$, Favarato $D$, Ramires J A. Vaccination against the influenza virus and mortality due to cardiovascular diseases in the city of São Paulo. Arq Bras Cardiol. 2009 Oct;93(4):395-9. doi: http://dx.doi.org/10.1590/S0066$782 \times 2009001000013$.

44. Committee on infectious diseases. Recommendations for prevention and control of influenza in children, 2013-2014. Pediatrics. 2013 Oct;132(4):e1089-104. doi: 10.1542/peds.2013-2377. PubMed PMID: 23999962.

45. Guan X, Yang W, Sun X, Wang L, Ma B, Li H, et al. Association of influenza virus infection and inflammatory cytokines with acute myocardial infarction. Inflamm Res. 2012 Jun;61(6):591-8. doi: 10.1007/s00011-012-0449-3. PubMed PMID: 22373653.

46. Liu IF, Huang CC, Chan WL, Huang PH, Chung CM, Lin SJ, et al. Effects of annual influenza vaccination on mortality and hospitalization in elderly patients with ischemic heart disease: a nationwide population-based study. Prev Med. 2012 Jun;54(6):431-3. doi: 10.1016/j.ypmed.2012.03.020 . PubMed PMID: 22504030

47. Macintyre CR, Heywood AE, Kovoor P, Ridda I, Seale H, Tan T et al. Ischaemic heart disease, influenza and influenza vaccination: a prospective case control study. Heart. 2013 Dec;99(24):1843-8. doi:10.1136/heartjnl-2013-304320. PubMed PMID: 23966030.

48. Kopel E, Klempfner R, Goldenberg I. Influenza vaccine and survival in acute heart failure. Eur J Heart Fail. 2014 Mar;16(3):264-70. doi: 10.1002/ejhf.14. PubMed PMID: 2464586.

49. Seo YB, Choi WS, Baek JH, Lee J, Song JY, Lee JS, et al. Effectiveness of the influenza vaccine at preventing hospitalization due to acute exacerbation of cardiopulmonary disease in Korea from 2011 to 2012. Hum Vaccin Immunother. 2014 Feb;10(2):423-7. doi: 10.4161/hv.26858. PubMed PMID: 24161939.

50. Hebsur S, Vakil E, Oetgen WJ, Kumar PN, Lazarous DF. Influenza and coronary 
artery disease: exploring a clinical association with myocardial infarction and analyzing the utility of vaccination in prevention of myocardial infarction. Rev Cardiovasc Med. 2014;15(2):168-75. PubMed PMID: 25051134.

51. Davis MM, Taubert K, Benin AL, Brown DW, Mensah GA, Baddour LM, et al. Influenza vaccination as secondary prevention for cardiovascular disease: a science advisory from the American Heart Association/American College of Cardiology. J Am Coll Cardiol. 2006 Oct 3;48(7):1498-502. Epub 2006 Sep 15. PubMed PMID: 17010820.

52. Centers for Disease Control and Prevention. Flu and heart disease \& stroke: people with heart disease and those who have had a stroke are at high risk of developing complications from influenza (the flu) [Internet]. 2014 [cited Aug 28]. Available from: http://www.cdc.gov/flu/heartdisease/

53. Centers for Disease Control and Prevention. Prevention and control of seasonal influenza with vaccines. Recommendations of the Advisory Committee on Immunization Practices--United States, 2013-2014. MMWR Recomm Rep. 2013 Sep 20;62(RR-07):1-43. PubMed PMID: 24048214.

54. Neuzil KM. Influenza vaccination in 2013-2014: achieving $100 \%$ participation. JAMA. 2013 Oct 23;310(16):1681-2. doi: 10.1001/jama.2013.279207. PubMed PMID: 24150465.

55. Rogers KC, Wallace JP, Foster SL, Finks SW. Annual influenza vaccination: offering protection beyond infection. South Med J. 2012 July;105(7):379-86. doi: 10.1097/SMJ.0b013e31824e8db6. PubMed PMID: 22766667.

56. Ortiz JR, Neuzil KM, Cooke CR, Neradilek MB, Goss CH, Shay DK. Influenza pneumonia surveillance among hospitalized adults may underestimate the burden of severe influenza disease. PLoS One. 2014 Nov 25;9(11):e113903. doi:10.1371/journal.pone.0113903. PubMed PMID: 25423025. 